\title{
LE PRINCIPALI IPOTESI SULL'ISOSTASIA ${ }^{* \prime !}$
}

\author{
C. Aquilina
}

Sin dal tempo di Newton si era notato il comportamento anomalo della deviazione della verticale nelle vicinanze di masse superficiali di notevoli dimensioni. Bouguer aveva segnalato il fenomeno e Maskelyne aveva riprese le considerazioni del precedente. Si era concluso sulla esigua densità delle Cordigliere rispetto a quella media terrestre.

Ma a Pratt, arcidiacono di City Deep, poi di Calcutta, si deve (1855) la ipotesi della presenza di una superficie di compensazione ad una certa profondità sotto il livello medio del mare.

Teoria di Pratt.

Secondo questo autore, che aveva a ragione criticato il procedimento seguito da Everest nel compensare la differenza trovata fra le latitudini astromiche e geodetiche delle stazioni di Kanliapur e Kaliana, della triangolazione indiana, era necessario trovare una spiegazione per questo scarto. Non riporteremo qui il ragionamento, le conclusioni, le dispute, cose tutte consacrate alla storia di questo campo della scienza e ci limiteremo a riportare $\mathbf{i}$ caratteri generali della teoria enunciata da questo scienziato.

Essa può essere espressa nella semplice forma seguente.

Ad una profondità ipotetica data, e da considerarsi costante, si trova una superficie al di sopra della quale le masse, per unità di area, (che su di essa insistono) sono in ogni punto uguali. È come asserire che, lungo la verticale, le montagne hanno subito una dilatazione, gli avvallamenti oceanici, una contrazione. È verificata dunque la legge di dipendenza della densità di ciascun blocco elementare, vedi fig. 1 , dalla sua altezza $h$ sul geoide o dalla sua profondita $p$ sotto la superficie marina.

Il difetto di densità segnalato nei blocchi tipo $1,2,3$, o l'eccesso di essa in quelli tipo $4,5,6,7$, rispetto a quella media che si riscontra in quelli tipo 8 , ha termine sulla superficie di compensazione.

(*) Comunicazione presentati al Convegno dell'Associazione Geofisica Italiama, tenuto a Roma il 10-1I giugno 1952. 
Il valore $H$, profondità sotto il geoide della superficie di compensazione, $\grave{e}$ arbitrario e va fissato, nei calcoli eventuali, in maniera da permettere il raggiungimento di risultati che giustifichino le anomalie dalle quali ebbe origine la teoria stessa.

Hayford, ammettendo l'ipotesi di una compensazione perfetta, secondo la teoria di Pratt, iniziò la riduzione delle osservazioni di tutta la rete gravimetrica degli Stati Uniti. Per facilitare le operazioni di calcolo costrui delle tavole di riduzione cbe furono poi riprese e, pur mantenendo lo schema principale di esse, integralmente rifatte dai nostri G. Cassinis, P. Dore e S. Ballarin.

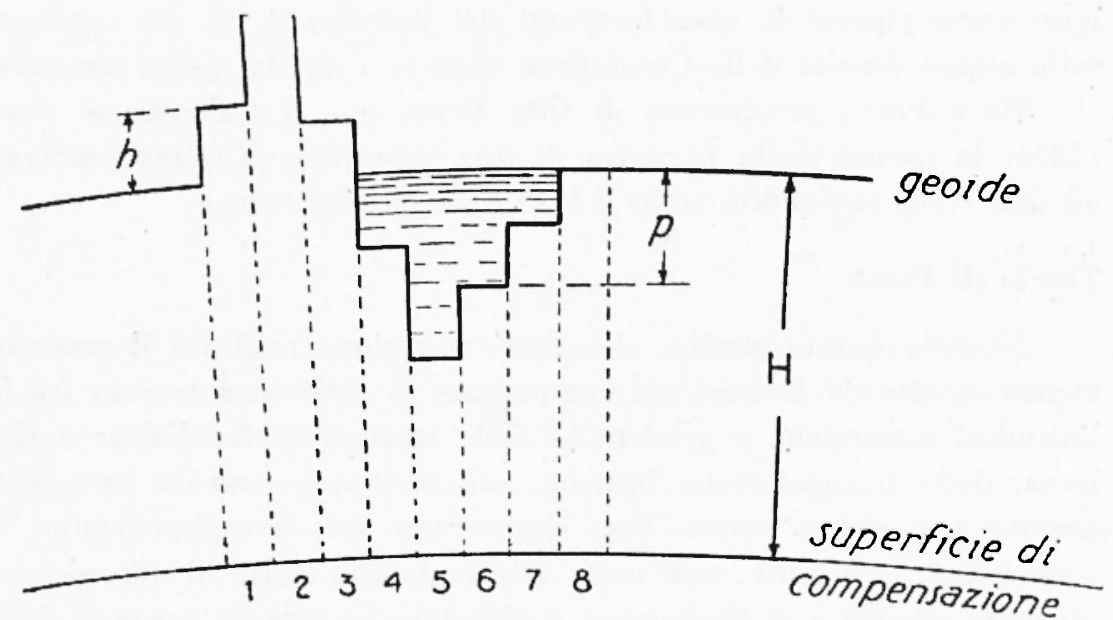

Fig. 1

Queste tavole sono, invero, quelle che hanno reso agevole la grande mole del lavoro di riduzione delle determinazioni gravimetriche, operazione, altrimenti, praticamente impossibile a portarsi a compimento.

Nel calcolo di queste riduzioni vanno tenute nel massimo conto le approssimazioni che possono essere raggiunte sia nella scelta dello schema di ripartizione in blocchi elementari, nelle regioni prossime e lontane, sia nella ricerca dei valori di $h$ e di $p$ e quindi degli accorgimenti da adottarsi per rendersi esenti dagli errori commessi per l'impossibilità di conoscere detti valori nelle regioni topograficamente meno note, o da ridurli al minimo possibile.

Osserviamo ( ${ }^{1}$ ) che nell'ipotesi di Pratt, considerata sotto il suo aspetto fisico, l'uguaglianza delle masse. dei blocchi elementari porta ad una disuguaglianza delle pressioni insistenti sulla superficie di 
compensazione, mentre sarebbe più logico attendersi un equilibrio idrostatico, che porta all'uguaglianza delle pressioni. Si dovrebbe fra l'altro, tener presente che l'accelerazione di gravità varia con la latitudine. E peraltro intuibile che negli strati profondi si sia realmente stabilito un equilibrio idrostatico ed inoltre all'aumentare delle profondità, con l'aumento della temperatura, c'è da aspettarsi una diminuzione della viscosità nelle masse.

Inoltre l'ipotesi del Pratt, seguita con rigore, vuole blocchi indipendenti, comportantisi indipendentemente ciascuno dai suoi vicini: è necessario dunque trascurare le reazioni laterali. Se si segue l'ipotesi di un equilibrio idrostatico è ovviamente necessario supporre, ad una certa profondità, l'eguaglianza delle pressioni: ciò comporta anche la necessità di limitare le reazioni laterali alle sole pressioni idrostatiche.

Un'altra osservazione va fatta, e del massimo interesse.

Senza voler entrare nel merito della rispondenza o meno con la realtà geologica primitiva dell'ipotesi trattata, che peraltro non soddisfa notoriamente $i$ punti di vista degli eminenti cultori di questo campo, dobbiamo ammettere che una rottura dell'equilibrio isostatico successiva, dovuta ad erosione o sedimentazione, o ad altro, non si sa come possa venire compensata.

Lo stesso Pratt ammetteva, d'altronde, che la sua ipotesi era approssimata.

E da osservare tuttavia che seguire l'ipotesi significa aspettarsi la compensazione in atto nelle formazioni più recenti $\left({ }^{1}\right)$. E notorio che si avvicina al vero il contrario: nelle più antiche piattaforme continentali si raggiunge la compensazione, nelle regioni ancora soggetto ad assestamento $\dot{e}$ invece estremamente improbabile che si abbia.

Ipotesi di Airy.

Quasi contemporaneamente alla pubblicazione della memoria di Pratt, riguardante la teoria esposta, l'astronomo Airy esprimeva concisamente il " suo schema ", di compensazione. Partendo dall'osservazione che uno spessore anche notevole della litosfera non poteva assicurare a questa una stabilità, per la viscosità delle roccie, e che quindi era illogico supporre che le escrescenze, riguardanti le alture, fossero sorrette dalle regioni circostanti, egli immaginava che i varî blocchi, affondando più o meno nel "sima " sottostante, per il principio di Archimede, subissero automaticamente la compensazione.

La densità media delle roccie doveva esser considerata costante 
e così quella del fluido magmatico sottostante. Questa concezione permetteva lenti movimenti verticali che in qualche modo portavano al susseguente ristabilimento della compensazione che aveva subito una rottura, dovuta ad un movimento orogenetico o ad altro.

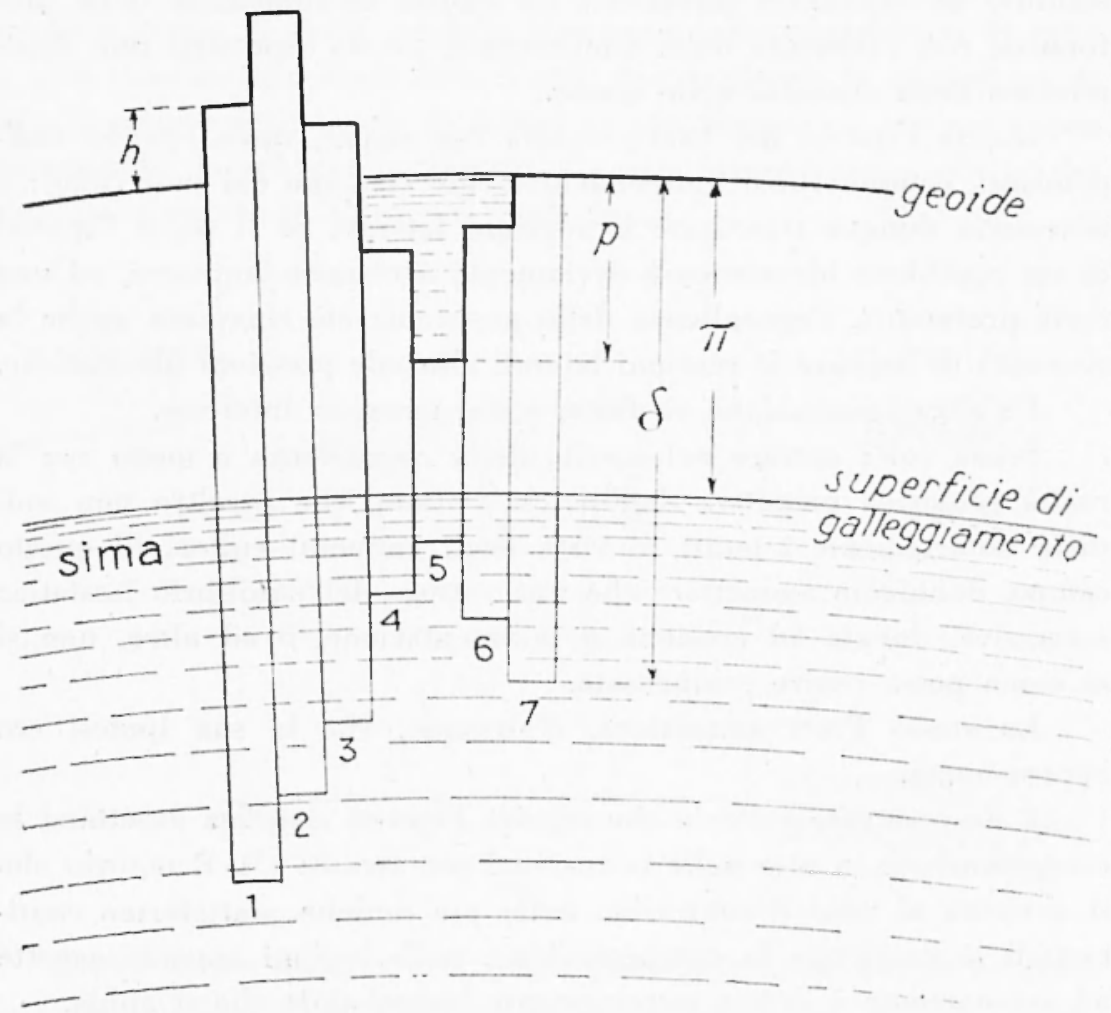

Fig. 2

Nella fig. 2 sono rappresentati schematicamente gcli aflondamenti dei varî blocchi nel sina: quelli continentali, n. $1,2,3$, di più di quello campione, indicato col n. 7, quelli oceanici, n. 4, 5, 6, di meno.

Naturalmente, come si è prima detto, va fissata la densità del sima; va fissata ancora l'altra incognita: la profondità II, rispetto al geoide, della superficie di galleggiamento. Questa ha peraltro notevole influenza sulla compensazione e malauguratamente, non si hanno indicazioni che possano guidarci ad un valore sia pure di largo orientamento.

Facciamo presente che anche nel trattare questa ipotesi, se a prima vista la possibilità del raggiungimento dell'equilibrio idrostatico, 
dei blocchi elementari, sembra intuitivo, la cosa non si presenta più cosi semplice ad un esame approfondito.

A parte il fatto che i singoli blocchi, per un sopravvenuto turbamento nell'equilibrio precedentemente raggiunto (per esempio per erosione o sedimentazione) dovreblero variare la loro posizione verticale, senza sulire l'influenza dei blocchi vicini, le stesse leggi del galleggiamento dovrebbero risentire, nella fase di assestamento, delle condizioni transitorie, anche per $\mathbf{i}$ blocchi vicini.

Le reazioni laterali, inoltre, per la convergenza delle verticali, dovrebbero dar luogo a risultanti verticali e, sotto la loro influenza, $i$ blocchi non dorreblero comportarsi da corpi perfettamente rigidi.

C'è anche da osservare che non è probabile che le basi di questi blocchi siano perfettamente rigide ed a contatto del sima fluido.

La concezione di Airy non premette alcuna ipotesi che possa essere contraria alle cognizioni greologiche e si limita ad ammettere che l'equilibrio isostatico si raggiunge, man mano, per il principio di Irchimede. Tutto sta a redere come si comporta la crosta terrestre per potersi assoggettare alla realizzazione di un vero e proprio galleggiamento di blocchi o, ciò che è lo stesso, di un comportamento schematizzabile in questo modo.

Intanto non ripugna il pensiero di un equilibrio isostatico interrotto, per esempio da una sedimentazione sopravvenuta, e quindi man mano ripristinato.

Rimane naturalmente da vedere quale sia il comportamento delle rocce alle temperature ed alle pressioni delle grandi profondità, per durate dell'ordine di millenni e millenni. Esperimenti ed interpretazioni si tentano per cercare in qualche modo di sollevare un velo del mistero impenetrato. Goguel $\left({ }^{2}\right)$ ad esempio, dichiara che le materie più plastiche, penetrando nei ruoti delle rocce più dure, ne mutano completamente le caratteristiche elastiche.

Nelle sue linee generali, dunque, l'ipotesi di Airy non è fuori delle possibilità reali, le concezioni semplificative dei calcoli, invece, come ad esempio l'ipotesi di costanza della densità, portano a conclusioni meno verosimili.

Ipotesi di Vening-Meinez. - Passiamo ora all'ipotesi enunciata da Vening-Meinez, che si differenzia dalle precedenti perché è un sistema di compensazione regionale che tiene conto delle reazioni laterali dei blocehi vicini. Le due precedenti ipotesi, quella di Pratt e quella di 
Airy, infatti, immaginano una compensazione strettamente verticale, localizzata ciò̀ al di sotto delle masse superficiali.

La correzione regionale invece presuppone la litosfera come una piastra piana, estesa indefinitamente, elastica, galleggiante, in equilibrio idrostatico, su uno strato, inferiore, plastico, a densità superiore.

Le escrescenze topografiche sovrapposte, al di sopra del livello del mare, causano una certa flessione, appunto per il loro peso, della piastra cbe è soggetta alle leggi dell'elasticità.

La compensazione dei " sovraccarichi topografici " avviene insomma per l'affondamento, ad essi proporzionale, della piastra nella sottostante massa plastica a densità superiore, tenuto opportuno conto però degli effetti laterali.

Velle regioni ricoperte dal mare, nelle quali si ha una topografia, rispetto al livello marino, negativa, si ha un qualche cosa di inverso. Si ha cioè compensazione per la presenza di masse più dense sollevantisi, come nel caso dell'ipotesi di Airy, che prendono il posto di quelle mancanti, della piastra, alla radice di essa.

Il problema, nei suoi aspetti analitici, viene impostato inmaginando una piastra indefinita, di opportuno spessore, assoggettata ad una pressione dovuta alla differenza delle densità tra piastra e massa marrmatica, per l'affondamento della detta piastra, ed in più, ad un peso concentrato che riguarda il sovraccarico dovuto alla topografia.

Il problema, di teoria dell'elasticità, risolto da Hertz, dà un'espressione che è funzione delle caratteristiche elastiche della piastra.

La deformazione della piastra dà luogo all'affondamento della ra. dice dello strato, in corrispondenza del sovraccarico. Questo affonda. mento va rapidamente smorzandosi man mano che ci si allontana dal punto di massimo carico, ma, oltrepassato il punto di influenza nulla, se ancora ci si allontana, si ha un sollevamento, con delle successive ondulazioni della superficie inferiore dello strato, fino ad una sparizione totale del fenomeno.

Il diagramma, che rappresenta queste deformazioni, viene preso come base per la ripartizione della compensazione, nel senso orizzontale: si ha pertanto un massimo al disotto dell'elemento disturbante $e_{\text {, }}$ man mano che da questo ci si allontana, si arriva alla compensazione nulla, in corrispondenza della deformazione nulla.

Per semplificare, l'autore non prende in considerazione le ondulazioni successive al punto di annullamento della principale, e, ad espressioni più complesse, sostituisce funzioni più semplici che dànno 
valori coincidenti, con quelli delle precedenti, in punti caratteristici del diagramma di deformazione.

Il prof. Vening-Meinez ha poi disposto la compensazione nel senso verticale in modo da poter trarre il massimo utile dalle citate tavole fondamentali di G. Cassinis, P. Dore e S. Ballarin le quali possono rappresentare, nel sistema di compensazione in esame, tavole per « regionalità nulla ".

Lo stesso autore afferma che la ripartizione della compensazione nel senso verticale non riposa su alcuna interpretazione fisica particolare e questo allo scopo di permettere il facile uso di altre tavole che si appoggaiano su principî fisici diversi.

Dal punto di vista geofisico l'ipotesi del Vening-Meinez ammette una partecipazione regionale al problema della compensazione ammettendo il gralleggiamento della piastra sovrastante sul magma. Si ha in fondo l'aggiunta, alla crosta terrestre, di una tensione che dovrebbe manifestarsi, nelle formazioni recenti, con una variazione di densità, per la trazione, ai margini dei blocchi che "scendono".

Lo squardo generale dato alle varie ipotesi mostra di per se come in effetti ciascuno schema tenda a giustificare le anomalie che si incon. trano. che le misure fatte, per diverse vie, accusano, con una legge generale. Ora sembra fuor di dubbio che una tale legge, se si riferisce ad un raggiunto equilibrio, non sarà verificata se non nelle regioni non più perturlaate da movimenti, nelle quali non si manifestano più fenomeni sismici e così via.

D'altra parte il risultato delle correzioni introdotte nelle determinazioni gravimetriche, veramente riduce notevolmente le anomalie e si dimostra ancora che l'influenza dell'ipotesi adottata nella correzione è assai piccola ( ${ }^{1}$ ). Questo fatto stareblue a dimostrare che l'ipotesi di una compensazione isostatica ad opportuna profondità, non è fuori dalla realtì.

Ora ferma restando la presenza delle eccezioni nelle regioni nelle quali si è lontani da un equilibrio definitivo, si può asserire che sembra logico accettare l'ipotesi di un equilibrio raggiunto, nei casi ad esempio nei quali ci si trova davanti a vecchie piattaforme continentali, lipotesi di un assestamento nella sua fase ultima, nei casi nei quali si ha da fare con regioni che pur non essendo in condizioni di sicura stabilità, sono lontane dalla fase di tormenti sismici ecc.

Ora nelle regioni che hanno raggiunto al completo l'equilibrio (forse nessuna) sembra convincente attenersi all'ipotesi di Airy se os- 
serviamo che non ripugna pensare ad una stabilizzazione, nel senso verticale, anche immaginando il blocco che, con la profondità varî le sue caratteristiche fisiche fino a raggiungere quelle del sima. E poiché raggiunta la compensazione non si debbono avere reazioni laterali (altrimenti significherebbe che l'equilibrio non è stato raggiunto) si può ben pensare ad un blocco formato da una pila di rocce cbe man mano con la profondità passano ad avere le caratteristiche del sima.

La seconda ipotesi, la più generale, che si riferisce al sistema di compensazione regionale potreble pur rispondere ad un criterio come quello or ora esposto. Osserviamo ancora cbe Vening-Meinez, con il suo sistema di compensazione, nell'ammettere un assestamento, implicitamente, dovrà ammettere cbe la pressione, dovuta al sovraccarico topografico faccia prevedere un'azione propagantesi nel magma, che durerà fino al raggiungimento dell'equilibrio isostatico.

E ovvio, per certo, che la comprimibilità del fluido del quale si tratta dovrà assorbire parte di quelle pressioni, ma, tenuto il dovuto conto delle distanze, ed a meno di un coefficiente di attutimento del fenomeno, ci appare verosimile clue un sovraccarico come l'Himalaja, ad esempio, debba dare, almeno sotto $i$ mari più vicini, una spinta verso l'alto secondo il principio di Pascal.

Il fenomeno potrebbe giustificare una maggiore compressione e quindi una maggiore densità, delle regioni submarine, cosa che ci arvicinerebbe per il punto in esame e sotto questo aspetto, al concetto teorico di distribuzione delle densità supposte da Pratt.

Concludendo questi brevi cenni sulle ipotesi principali finora formulate sembra logico sottolineare l'apporto veramente imponente dato da varî illustri scienziati che hanno trattato il problema sia sotto lo aspetto teorico che sotto quello applicato, ma d'altra parte, né se lo sono nascosto gli stessi autori, le difficoltà da superare sono tali che sarebbe auspicabile, avere molti altri elementi a disposizione per poter parlare di teoria che si avvicini di più alla realtà dei fatti.

Roma - Istituto di Geofisica Mineraria della Facollà di Ingegne. ria dell'Università - Giugno 1952.

\section{RIASSUNTO}

L'Autore richiama le tre principali ipotesi sull'isostasia, e ciò̀ quella dovuta a Pratt, quella di Airy e quella di Vening-Meinez. Nel farne il confronto e la critica propone, tra laltro, di prendere in. 
considerazione le eventuali spinte, secondo il principio di Pascal, dovute alle azioni dei pesi dei forti dislivelli topografici.

\section{SUMMARY}

The author revieus the three principal hypotheses concerning isostasy; numely those of Pratt, Airy and Vening-Meinez. In making the comparison and proposing a criticism, the principle of Pascal concerning the possible effect of the weights of the substantial topographical inequalities has been taken into consideration.

\section{BIBLIOGRAFIA}

(1) Lfjay Pierre R. P., Développements modernes de la gravimetrie. Paris, Gauthier, Villars 1947.

(2) Gocuel J.: Introduction à l'étude mecanique des déformations de l'écorce ierrestre. Paris 1943-1948.

(3) Cassinis G. - Done P. - Ballarin S., Tavole fondamentali per la riduzione dei valori osservati della gravità. Pavia 1937.

(4) GiLatee B. L., Gravily Reductions and the Figure of the Earth. P. 323 Gerlands Beitrage zur Geophisik. Leipzig 1938, Band. 53.

(i) Meinez.Vexing F. A.s Tables Fondamentales pour la Réduction Isostatique Regional. Bulletin Géodésique, p. 711, n. 63, 1939. 